\title{
UPPER AND LOWER FUNCTIONS OF PLANE BROWNIAN ANGLES
}

\author{
E. A. Dorofeev
}

UDC 519.2

One of the most rapidly growing fields in the theory of random processes is asymptotics of multidimensional Wiener functionals, including such functionals as winding angles or radius-vectors of planar processes. We present a new method yielding a large number of results on upper and lower functions for such functionals. Bibliography: 23 titles.

At present, problems of the geometry of multidimensional Brownian curves and close problems for random walks are widely studied. In recent years, important new results have been obtained on the asymptotic behaviour of functionals of winding-angle type for the plane Brownian motion $Z_{t}, t \geqslant 0$ (see, e.g., [23, 15, 17]). Various limit theorems for the winding angle $\theta_{t}$ were obtained; most of them deal with the winding of the process $Z$ around the origin or around another Brownian particle, which is independent of the first one. Here, [14] and [23] should be mentioned. Interesting results for the asymptotics of the number of turns of the radius vector of a three-dimensional Wiener particle about some curves have also been obtained (see e.g., $[6])$.

Let us turn to the intuitive notion of the winding angle of a two-dimensional (continuous) Brownian path around the origin. ${ }^{1}$ Obviously, $\theta_{t}$ has symmetric continuous distribution at any moment $t>0$. We present a rigorous definition of the $\theta$-process somewhat later.

The first result, obtained already by F. Spitzer [21], may be regarded as "the law of large numbers" for $\theta_{t}$ :

$$
\frac{2}{\log t} \theta_{t} \stackrel{L}{\rightarrow} C_{1}, \quad t \rightarrow \infty,
$$

where $C_{1}$ denotes the standard Cauchy distribution with parameter 1 , and means convergence in probability.

More precise asymptotics of $\theta_{t}$ were obtained by J. Pitman and M. Yor (see $[17,18]$ ) and later by P. Messulam and Yor [16], who considered "small" and "large" angles $\theta$, namely, the components of the winding process in and outside a small neighborhood of the origin. They considered the asymptotics of the Fourier transform of the following pair of random variables:

$$
\left(\frac{2}{\log t} \int_{0}^{t} \mathbb{I}\left(\rho_{s}<\varepsilon\right) d \theta_{s}, \frac{2}{\log t} \int_{0}^{t} \mathbb{I}\left(\rho_{s}>\varepsilon\right) d \theta_{s}\right),
$$

where $\rho_{s}=\left\|Z_{s}\right\|, \varepsilon>0$, and $\mathbb{I}(\cdot)$ denotes the indicator of a random event. (This notation is preserved in what follows.) They showed that

$$
\lim _{t \rightarrow \infty} \mathbf{E} \exp \left(\frac{2 \mu i}{\log t} \int_{0}^{t} \mathbb{I}\left(\rho_{s}<\varepsilon\right) d \theta_{s}+\frac{2 \lambda i}{\log t} \int_{0}^{t} \mathbb{I}\left(\rho_{s}>\varepsilon\right) d \theta_{s}\right)=\left(\cosh \lambda+\frac{|\mu|}{\lambda} \sinh \lambda\right)^{-1},
$$

$\mu, \lambda \in \mathbb{R}$ (see [16, p. 356] or [15]). Thus, the limit does not depend on $\varepsilon$.

In particular, it follows from (2) that the Fourier transform of the limit distribution of the variable

$$
\frac{2}{\log t} \int_{0}^{t} \mathbb{I}\left(\rho_{s}>\varepsilon\right) d \theta_{s}
$$

\footnotetext{
${ }^{1}$ Here and below, we suppose that the Brownian curve does not pass through the point $(0,0)$. It is usually assumed that $Z_{0}=(1,0)$. If we put $Z_{0}=(0,0)$, then the Brownian particle turns infinitely many times around the origin during an arbitrary short period of time after the start and hence the winding angle is not well defined.
}

Translated from Zapiski Nauchnykh Seminarov POMI, Vol. 228, 1996, pp. 111-134. Original article submitted December 10, 1995. 
is equal to $\operatorname{sech} \lambda$, and, consequently, the limit distribution has density $\frac{1}{2} \operatorname{sech} \frac{\pi x}{2}, x \in \mathbb{R}$ (see $[8$, p. 476 , Eq. 10]). Note that it is an infinitely divisible distribution (see [8, p. 537]).

Messulam and Yor answered a natural question arising from the fact that the limit distribution (2) does not depend on $\varepsilon$ (see [16, p. 362]). They showed that the limit distribution of $\int_{0}^{t} \mathbb{I}\left(\rho_{s} \in\left[\varepsilon_{1}, \varepsilon_{2}\right]\right) d \theta_{s}$, $0<\varepsilon_{1}<\varepsilon_{2}$, as $t \rightarrow \infty$ is as follows:

$$
\sqrt{\frac{2}{\log t}} \int_{0}^{t} \mathbb{I}\left(\rho_{s} \in[\varepsilon, 1]\right) d \theta_{s} \stackrel{L}{\rightarrow} N(0,1) \sqrt{l_{0}^{\sigma_{1}}|\log \varepsilon|} .
$$

Here, $l_{0}^{\sigma_{1}}$ denotes the local time at the zero point of the standard linear Brownian motion $\beta$ up to time $\sigma_{1}$ of hitting the point 1 , and $N(0,1)$ is a standard Gaussian variable independent of $\beta$. We remind the reader that for any positive $t$ we have

$$
l_{0}^{t}=\lim _{\delta \rightarrow 0} \frac{1}{\delta} \int_{0}^{t} \mathbb{I}\{\beta \in[0, \delta]\} d t
$$

a.s. (see, e.g., $[1$, p. 8]).

Finally, J. Bertoin and W. Werner [4] and later Z. Shi [20] obtained results on the upper and lower functions for certain functionals of the winding type. Our aim here is to obtain a whole series of such results.

Let us turn to the basic notation. Let $Z_{s}=\left(X_{s}, Y_{s}\right), s \geqslant 0$, be the standard planar Brownian motion.

We define the process $\theta_{s}$ as a solution of the Ito stochastic equation

$$
d \theta_{s}=\frac{X_{s} d Y_{s}-Y_{s} d X_{s}}{\rho_{s}^{2}}, \quad \theta_{0}=0
$$

The solution exists if the modulus $\rho_{\mathrm{s}}$ of the process $Z_{s}$ is a.s. bounded away from zero, $\forall s>0$. This condition is satisfied if $Z_{0} \neq 0$.

We study the following functionals:

$$
\begin{aligned}
& \Phi_{1}(t)=\int_{0}^{t} \mathbb{I}\left(\rho_{s} \in\left[\varepsilon_{1}, \varepsilon_{2}\right]\right) d \theta_{s}, \\
& \Phi_{2}(t)=\int_{0}^{t} \mathbb{I}\left(\rho_{s}>\varepsilon\right) d \theta_{s}, \\
& \Phi(t)=\int_{0}^{t} d \theta_{s}=\theta_{t} .
\end{aligned}
$$

The continuous local martingales

$$
\frac{X_{s} d X_{s}+Y_{s} d Y_{s}}{\rho_{s}^{2}} \text { and } \frac{X_{s} d Y_{s}-Y_{s} d X_{s}}{\rho_{s}^{2}}
$$

which are respectively equal a.s. to the process $\log \rho_{s}$ and to the winding process $\theta_{s}$ of the Wiener process $Z$ (see [17]), can be written in terms of two independent linear Wiener processes $\left(\beta_{C_{\imath}}, \gamma_{C_{\imath}}\right)$ (see $[7$, p. 443$]$ and $[16$, p. 356]) after the time-change

$$
C_{t}=\int_{0}^{t} \rho_{s}^{-2} d s
$$


More precisely, for all $\varepsilon_{1}>\varepsilon_{2}>0$ and $\varepsilon>0$, we have a.s.

$$
\begin{aligned}
\Phi_{1}(t) & =\int_{0}^{C_{t}} \mathbb{I}\left(\beta_{s} \in\left[\log \varepsilon_{1}, \log \varepsilon_{2}\right]\right) d \gamma_{s}, \\
\Phi_{2}(t) & =\int_{0}^{C_{t}} \mathbb{I}\left(\beta_{s}>\log \varepsilon\right) d \gamma_{s}, \\
\Phi(t) & =\int_{0}^{C_{t}} d \gamma_{s} .
\end{aligned}
$$

Furthermore, for all positive $r$, we have

$$
C_{T(r)}=\sigma_{\log r} \text { a.s., }
$$

where $\sigma_{s}=\inf \left(u \geqslant 0: \beta_{u}=s\right), T(r)=\min \left(s \geqslant 0: \rho_{s}=r\right)$, and

$$
\frac{\log T(r)}{2 \log r} \rightarrow 1, \quad r \rightarrow \infty \text { a.s. }
$$

(see [16, p. 355]). Using (4), we prove several results of the type of the "law of the iterated logarithm" (LII) for the winding process $\theta$. More precisely, we prove theorems on upper and lower functions of $\theta$. We obtain some estimate for the sojourn times

$$
\int_{0}^{\sigma_{\log r}} \mathbb{I}\left(\beta_{s} \in\left[\log \varepsilon_{1}, \log \varepsilon_{2}\right]\right) d s, \quad \int_{0}^{\sigma_{\log r}} \mathbb{I}\left(\beta_{s}>\log \varepsilon_{1}\right) d s, \text { etc. }
$$

and substitute the result in the standard LIL for the linear Brownian motion $\gamma$ or in the Fristedt formula for liminf $\sup _{0<s<t}\left|\gamma_{s}\right|$ (see [9, Theorem 11.6]).

Such an approach allows us to obtain many more results for the upper and lower functions for the mentioned functionals than by using precise estimates for the Laplace transforms of the corresponding distributions (see [20]) or by applying explicit formulas for them, which is possible only for very special functionals.

Let $I=I(t), t \geqslant 0$, be a positive random functional. We call $U_{1}$ and $D_{1}$ (respectively, $U_{2}$ and $D_{2}$ ) the upper and the lower functions of the first and (respectively, second) type for the functional $I$ if a.s.

$$
\begin{gathered}
\limsup _{t \rightarrow \infty}|I(t)| / U_{1}(t)=0, \quad \underset{t \rightarrow \infty}{\limsup }|I(t)| / D_{1}(t)=\infty, \\
\liminf _{t \rightarrow \infty} \frac{\sup _{0 \leqslant s \leqslant t}|I(t)|}{U_{2}(t)}=0, \quad \liminf _{t \rightarrow \infty} \frac{\sup _{0 \leqslant s \leqslant t}|I(t)|}{D_{2}(t)}=\infty .
\end{gathered}
$$

Lemma 1 (see $\left[11\right.$, p. 1626]). There exists a linear Brownian motion $\gamma^{*}$ on the probability space generated by the processes $\beta$ and $\gamma$ such that 2.s. for all positive $t$ we have

$$
\int_{0}^{C_{t}} \mathbb{I}\left(\beta_{s} \in\left[\log \varepsilon_{1}, \log \varepsilon_{2}\right]\right) d \gamma_{s}=\int_{0}^{r_{1}\left(C_{t}\right)} d \gamma_{s}^{*}=\gamma^{*}\left(\tau_{1}\left(C_{t}\right)\right),
$$

where

$$
\tau_{1}(t)=\tau_{\left[\log e_{1}, \log \varepsilon_{2}\right]}^{[0, t]}=\int_{0}^{t} \mathbb{I}\left(\beta_{s} \in\left[\log \varepsilon_{1}, \log \varepsilon_{2}\right]\right) d s
$$

is independent of $\gamma^{*}$.

Note that similar relations hold for $\int_{0}^{C_{t}} d \gamma_{s}$, with $\tau_{1}$ replaced by

$$
\tau_{1}(t)=\tau_{(-\infty, \infty)}^{[0, t]}
$$

and, respectively, for $\int_{0}^{C_{t}} \mathbb{I}\left(\beta_{s} \in\left[\log \varepsilon_{1}, \log \varepsilon_{2}\right]\right) d \gamma_{s}$.

Let $\alpha>1$. Define $R_{k}=\alpha^{k}, k \in \mathbb{N}$. 
Lemma 2. Let $F$ be an increasing function and let $w$ be a standard linear Brownian motion starting at zero. Assume that

$$
\sigma_{r}=\min \left\{s>0: \beta_{s}=r\right\},
$$

where $\beta$ is another standard linear Brownian motion independent of $w$. If

then the random events

$$
\sum_{k} \mathbf{P}\left(\sup _{0 \leq s \leq \sigma_{R_{k}}}|w(s)| \geqslant F\left(R_{k}\right)\right)=\infty
$$

hold infinitely often a.s.

$$
A_{k}=\left\{\sup _{0 \leq s \leq \sigma_{R_{k}}}|w(s)| \geqslant F\left(R_{k}\right)\right\}
$$

Proof. Consider the sequence of $\sigma$-algebras

$$
\mathcal{F}_{t}=\sigma\left(\sigma\{w(s), s \geqslant 0\} \times \sigma\left\{\beta_{s}, 0 \leqslant s \leqslant t\right\}\right) .
$$

Events $A_{k}$ are obviously $\mathcal{F}_{\sigma_{R_{k}}}$-measurable.

We show that under the assumptions of Lemma 2, we have

$$
\sum_{k} \mathbf{P}\left(\sup _{0 \leq \leq \leq \sigma_{R_{k}}}|w(s)| \geqslant F\left(R_{k}\right) \mid \mathcal{F}_{\sigma_{R_{k-1}}}\right)=\infty \text { a.s. }
$$

By the Borel-Cantelli-Lévy lemma (see [22, p. 553]), this implies the assertion of Lemma 2.

Set

$$
\mathcal{I}_{k}=\min \left(\min \left\{s \geqslant \sigma_{R_{k-1}}: \beta_{s}=0\right\}, \sigma_{R_{k}}\right) .
$$

We have a.s.

$$
\begin{aligned}
& \mathbf{P}\left(\sup _{0 \leq s \leq \sigma_{R_{k}}}|w(s)| \geqslant F\left(R_{k}\right) \mid \mathcal{F}_{\sigma_{R_{k-1}}}\right) \\
& \geqslant \mathbf{P}\left(\sup _{\tau_{k} \leq s \leq \sigma_{R_{k}}}|w(s)| \geqslant F\left(R_{k}\right) \mid \mathcal{F}_{\sigma_{R_{k-1}}}\right) \\
& \geqslant \mathbf{P}\left(\exists s \in\left[\sigma_{R_{k-1}}, \sigma_{R_{k}}\right]: \beta_{s}=0, \sup _{\mathfrak{I}_{k}<s \leq \sigma_{R_{k}}}|w(s)| \geqslant F\left(R_{k}\right) \mid \mathcal{F}_{\sigma_{R_{k-1}}}\right) \\
& =\mathrm{E}\left[\mathbb{I}\left(\inf _{\sigma_{R_{k-1}} \leq \unlhd \leq \mathcal{T}_{k}}\left|\beta_{s}\right|=0\right) \mathbb{I}\left(\sup _{\mathfrak{T}_{k}<s \leq \sigma_{R_{k}}}|w(s)| \geqslant F\left(R_{k}\right)\right) \mid \mathcal{F}_{\sigma_{R_{k-1}}}\right] \\
& =\mathbf{E}\left[\mathbb{I}\left(\inf _{\sigma_{R_{k-1}}<s \leq \mathcal{I}_{k}}\left|\beta_{s}\right|=0\right) \mathbf{P}\left(\sup _{\mathcal{T}_{k}<s \leq \sigma_{R_{k}}}|w(s)| \geqslant F\left(R_{k}\right) \mid \mathcal{F}_{\boldsymbol{I}_{k}}\right) \mid \mathcal{F}_{\sigma_{\boldsymbol{R}_{k-1}}}\right] \text {. }
\end{aligned}
$$

Note that by the definition of the moment $\mathfrak{I}_{k}$, we have

$$
\mathfrak{I}_{k}<\sigma_{R_{k}} \Rightarrow \beta_{\mathfrak{T}_{k}}=0 \text {. }
$$

Using the strong Markov property of the Wiener process, we get

$$
\begin{aligned}
& \mathbf{P}\left(\sup _{\mathcal{I}_{k}<s \leq \sigma_{R_{k}}}|w(s)| \geqslant F\left(R_{k}\right) \mid \mathcal{F}_{\boldsymbol{T}_{k}}\right) \\
& \geqslant \mathbf{P}\left(\sup _{\mathcal{I}_{k}<s \leq \sigma_{R_{k}}}|w(s)| \geqslant F\left(R_{k}\right),\left|w\left(\mathcal{I}_{k}\right)\right|<F\left(R_{k}\right)\right) \\
& \geqslant \mathbf{P}\left(\sup _{0<s \leq \sigma_{R_{k}}} w(s) \geqslant F\left(R_{k}\right)\right) \\
& \geqslant \frac{1}{2} \mathbf{P}\left(\sup _{0<s \leq \sigma_{R_{k}}}|w(s)| \geqslant F\left(R_{k}\right)\right) .
\end{aligned}
$$


Consequently, (8) implies

$$
\begin{aligned}
& \mathbf{P}\left(\sup _{0 \leq s \leq \sigma_{R_{k}}}|w(s)| \geqslant F\left(R_{k}\right) \mid \mathcal{F}_{\sigma_{R_{k-1}}}\right) \\
& \geqslant \frac{1}{2} \mathbf{P}\left(\sup _{0<s \leq \sigma_{R_{k}}}|w(s)| \geqslant F\left(R_{k}\right)\right) \\
& \quad \quad \times \mathbf{P}\left(\exists s \in\left[\sigma_{R_{k-1}}, \sigma_{R_{k}}\right]: \beta_{s}=0 \mid \mathcal{F}_{\sigma_{R_{k-1}}}\right) \\
& =\frac{\alpha-1}{2 \alpha} \mathbf{P}\left(\sup _{0<s \leq \sigma_{R_{k}}}|w(s)| \geqslant F\left(R_{k}\right)\right)
\end{aligned}
$$

and the series

$$
\sum_{k} \mathbf{P}\left(\sup _{0 \leq s \leq \sigma_{R_{k}}}|w(s)| \geqslant F\left(R_{k}\right) \mid \mathcal{F}_{\sigma_{R_{k-1}}}\right)
$$

diverges a.s. The proof of Lemma 2 is complete.

Lemma 3. (a) Let $\beta$ be the above linear Brownian motion and let $l_{0}^{t}$ be its local time at zero up to the moment $t$. If

$$
\sum_{k} \mathrm{P}\left(\sup _{\substack{0 \leq s \leq l_{0}^{R_{k}}}}|w(s)| \geqslant F\left(R_{k}\right)\right)=\infty,
$$

then the random events

$$
A_{k}=\left\{\sup _{0 \leq s \leq I_{0}^{\sigma_{R_{k}}}}|w(s)| \geqslant F\left(R_{k}\right)\right\}
$$

hold infinitely often a.s.

(b) Let

$$
\tau_{2}(t)=\int_{0}^{t} \mathbb{I}\left(\beta_{s} \in[\log \varepsilon, \infty)\right) d s
$$

If

$$
\sum_{k} \mathbf{P}\left(\sup _{0 \leq s \leq \tau_{2}\left(\sigma_{R_{k}}\right)}|w(s)| \geqslant F\left(R_{k}\right)\right)=\infty,
$$

then the random events

$$
A_{k}=\left\{\sup _{0 \leq s \leq \tau_{2}\left(\sigma_{R_{k}}\right)}|w(s)| \geqslant F\left(R_{k}\right)\right\}
$$

hold infinitely often a.s.

Proof. To prove assertions (a) and (b), replace $w(s)$ by $w\left(l_{0}^{s}\right)$ and by $w\left(\tau_{2}(s)\right)$, respectively, everywhere in the proof of Lemma 2.

Corollary 1. Let $F^{(1)}, F^{(2)}$, and $F^{(3)}$ be increasing functions such that lim $\sup _{t} F^{(i)}(\alpha t) / F^{(i)}(t) \leqslant \alpha$ for every $\alpha$ greater than one. Let the series

$$
\begin{aligned}
& \sum_{k} \mathbf{P}\left(\sup _{0 \leq s \leq \sigma_{R_{k}}}|w(s)| \geqslant F^{(1)}\left(R_{k}\right)\right), \\
& \sum_{k} \mathbf{P}\left(\sup _{0 \leq s \leq i_{0}^{\sigma R_{k}}}|w(s)| \geqslant F^{(2)}\left(R_{k}\right)\right), \\
& \sum_{k} \mathbf{P}\left(\sup _{0 \leq s \leq \tau_{2}\left(\sigma_{R_{k}}\right)}|w(s)| \geqslant F^{(3)}\left(R_{k}\right)\right)
\end{aligned}
$$


converge (respectively, diverge) for every $\alpha$ greater than one.

Then we have a.s.

$$
\begin{aligned}
& \limsup \frac{\sup _{0} \leq s \leq \sigma_{R}|w(s)|}{F^{(1)}(R)} \leqslant 1 \quad(\geqslant 1), \\
& \limsup _{R} \frac{\sup _{0 \leq s \leq l_{0}^{\sigma R}}|w(s)|}{F^{(2)}(R)} \leqslant 1 \quad(\geqslant 1), \\
& \underset{R}{\limsup \frac{\sup _{0 \leq s \leq \tau_{2}\left(\sigma_{R}\right)}|w(s)|}{F^{(3)}(R)}} \leqslant 1 \quad(\geqslant 1) .
\end{aligned}
$$

Proof. Let series (10) converge. The case where (10) diverges is completely similar. It follows from the Borel-Cantelli lemma and Lemmas 2 and 3 that a.s.

$$
\begin{gathered}
\limsup \frac{\sup _{0 \leq s \leq \sigma_{R_{k}}}|w(s)|}{F^{(1)}\left(R_{k}\right)} \leqslant 1, \\
\limsup _{k} \frac{\sup _{0 \leq s \leq I_{0} R_{k}}|w(s)|}{F^{(2)}\left(R_{k}\right)} \leqslant 1, \\
\sup _{0 \leq s \leq \tau_{2}\left(\sigma_{R_{k}}\right)|w(s)|} \leqslant 1 .
\end{gathered}
$$

We prove the required statement for the first upper limit (the second and third limits are considered in a similar way).

Assume the contrary. Let

$$
\underset{k}{\limsup } \frac{\sup _{0 \leq s \leq \sigma_{R_{k}}}|w(s)|}{F^{(1)}\left(R_{k}\right)} \leqslant 1,
$$

but

$$
\underset{R}{\limsup } \frac{\sup _{0 \leq s \leq \sigma_{R}}|w(s)|}{F^{(1)}(R)}>1+\delta, \quad \delta>0 .
$$

Consequently, for every fixed $\alpha$ greater than one there exist infinitely many natural numbers $k$ such that the moments

$$
\left.t_{k}=\min \left\{s \in \mid \alpha^{k}, \alpha^{k+1}\right]: \frac{\sup _{0} \leq s \leq \sigma_{t_{k}}|w(s)|}{F^{(1)}\left(t_{k}\right)} \geqslant 1\right\}
$$

exist. The moments $t_{k}$ are obviously the Markov moments, and hence we have

$$
\left|w\left(\alpha^{k+1}\right)\right| \geqslant\left|w\left(t_{k}\right)\right|
$$

infinitely often because the random variables $\operatorname{sign}\left(w\left(\alpha^{k+1}\right)-w\left(t_{k}\right)\right)$ do not depend on $\mathcal{F}_{t_{k}}$. Consequently,

$$
\begin{aligned}
\limsup & \frac{\sup _{0} \leq s \leq \sigma_{\alpha^{k}}|w(s)|}{F^{(1)}\left(\alpha^{k}\right)} \geqslant K \limsup \frac{\sup _{0} \leq s \leq \sigma_{\alpha^{k}}|w(s)|}{F^{(1)}\left(\alpha^{k-1}\right)} \\
& \geqslant K \limsup _{k} \frac{\sup _{0 \leq s \leq \sigma_{c_{k}}}|w(s)|}{F^{(1)}\left(\alpha^{k-1}\right)} \\
& \geqslant K \operatorname{lim\operatorname {sup}} \frac{\sup _{0 \leq s \leq \sigma_{t_{k}}}|w(s)|}{F^{(1)}\left(t_{k}\right)} \geqslant K(1+\delta),
\end{aligned}
$$

where

$$
K=\liminf _{t} F^{(1)}(t) / F^{(1)}(\alpha t) \geqslant 1 / \alpha .
$$


Choosing $\alpha$ so that $1<\alpha<1+\delta$, we get

$$
\underset{k}{\limsup } \frac{\sup _{0} \leq s \leq \sigma_{\alpha^{k}}|w(s)|}{F^{(1)}\left(\alpha^{k}\right)}>1
$$

which contradicts (11). The relation

$$
\underset{k}{\limsup } \frac{\sup _{0 \leq s \leq \sigma_{\alpha^{k}}}|w(s)|}{F^{(1)}\left(\alpha^{k}\right)} \geqslant 1
$$

obviously implies that

$$
\underset{R}{\limsup } \frac{\sup _{0 \leq s \leq \sigma_{R}}|w(s)|}{F^{(1)}(R)} \geqslant 1 .
$$

The corollary is proved.

Now we can prove the following result.

Theorem 1 (Bertoin-Werner [4]). Let $F$ be an increasing function such that $F(x) / x \rightarrow \infty$. Then we have a.s.

$$
\underset{R}{\limsup } \frac{\left|w\left(\sigma_{R}\right)\right|}{F(R)}=0 \text { or } \infty
$$

depending on whether the integral

$$
\int_{0}^{\infty} d x / F(x)
$$

converges or diverges.

Proof. Without loss of generality, we may impose the additional condition

$$
\underset{t}{\limsup } F(\alpha t) / F(t) \leqslant \alpha, \quad \forall \alpha>1
$$

Indeed, the integral in the statement of the theorem converges if and only if the series

$$
\sum_{k=.}^{\infty} \frac{\alpha^{k}}{F\left(\alpha^{k}\right)}
$$

converges ( $\alpha>1$ is assumed to be fixed).

Furthermore, if series (R) converges, then there exists an increasing function $F^{\prime} \leqslant F$ such that a similar series with $F$ replaced by $F^{\prime}$ also converges and

$$
\limsup _{t} F^{\prime}(\alpha t) / F^{\prime}(t) \leqslant \alpha .
$$

Similarly, if (R) diverges, then there exists a function $F^{\prime} \geqslant F$ having the same property and satisfying (S).

To prove this, choose any increasing function $G$ satisfying $(S)$ and such that the corresponding series (R) converges (respectively, diverges); then we construct a "mixed" increasing series $F_{n}^{\prime}$ consisting of elements of the union $\left\{F_{n}, n \in \mathbb{N}\right\} \cup\left\{G_{n}, n \in \mathbb{N}\right\}$. The corresponding function $F^{\prime}$ is the required one.

Lemma 4. We have

$$
\mathbf{P}\left(\sup _{0 \leq s \leq \sigma_{r}} w(s) \geqslant y\right)=O(r / y), \quad r, y \rightarrow \infty, r / y \rightarrow 0
$$


Proof. Let us use relations 2.2 .1 and 5.0.2 of [1]:

$$
\begin{aligned}
& \mathbf{P}\left(\sup _{0 \leq s \leq t} w(s) \geqslant y\right)=\operatorname{Erfc}(y / \sqrt{2 t})=\frac{2}{\sqrt{\pi}} \int_{y / \sqrt{2 t}}^{\infty} e^{-z^{2}} d z, \\
& \mathbf{P}\left(\sigma_{r}<t\right)=\frac{r}{\sqrt{2 \pi}} \int_{0}^{t} \frac{e^{-r^{2} / 2 s}}{s^{3 / 2}} d s .
\end{aligned}
$$

The independence of $w$ and $\sigma_{r}=\min \left\{s>0: \beta_{s}=r\right\}$ implies that

$$
\begin{aligned}
& \mathbf{P}\left(\sup _{0 \leq s \leq \sigma_{r}} w(s) \geqslant y\right) \\
& =\frac{r}{\sqrt{2 \pi}} \int_{0}^{\infty} \operatorname{Erfc}(y / \sqrt{2 t}) \frac{e^{-r^{2} / 2 t}}{t^{3 / 2}} d t=\frac{r \sqrt{2}}{\sqrt{\pi}} \int_{0}^{\infty} \operatorname{Erfc}(y v / \sqrt{2}) e^{-r^{2} v^{2} / 2} d v \\
& =\frac{2 r \sqrt{2}}{\pi} \int_{0}^{\infty} e^{-r^{2} v^{2} / 2} d v \int_{y v / \sqrt{2}}^{\infty} e^{-z^{2}} d z=\frac{2 r \sqrt{2}}{\pi} \int_{0}^{\infty} e^{-z^{2}} d z \int_{0}^{z \sqrt{2} / y} e^{-r^{2} v^{2} / 2} d v \\
& =\frac{2}{\sqrt{\pi}} \int_{0}^{\infty} e^{-z^{2}} d z \sqrt{\frac{2}{\pi}} \int_{0}^{z \sqrt{2} r / y} e^{-x^{2} / 2} d u=O(r / y), \quad r, y \rightarrow \infty, r / y \rightarrow 0 .
\end{aligned}
$$

The lemma is proved.

Now let us return to the proof of Theorem 1. Fix an $\alpha$ greater than one and define $R_{k}=\alpha^{k} k \in \mathbb{N}$. Since $F(x) / x \rightarrow \infty$ and

$$
\mathbf{P}\left(\sup _{0 \leq s \leq \sigma_{r}} w(s) \geqslant y\right) \leqslant P\left(\sup _{0 \leq s \leq \sigma_{r}}|w(s)| \geqslant y\right) \leqslant 2 P\left(\sup _{0 \leq s \leq \sigma_{r}} w(s) \geqslant y\right),
$$

we see that for any positive $c$ the series

$$
\sum_{k} P\left(\sup _{0 \leq s \leq \sigma_{R_{k}}}|w(s)| \geqslant c F\left(R_{k}\right)\right)
$$

converges (respectively, diverges) simultaneously with series $(R)$. Using Corollary 1, we complete the proof of Theorem 1.

Corollary 2. Let $f$ be an increasing function such that

$$
\forall \alpha>1: \limsup _{t} f(\alpha t) / f(t) \leqslant \alpha .
$$

Then

$$
\underset{r}{\limsup } \frac{\left|w\left(\sigma_{\log r}\right)\right|}{\log r f(r)}=0 \text { or } \infty \text { a.s. }
$$

depending on whether the integral

$$
\int_{0}^{\infty} \frac{d x}{x \log x f(x)}
$$


converges or diverges.

Proof. Theorem 1 implies that

$$
\underset{r}{\limsup } \frac{\left|w\left(\sigma_{\log r}\right)\right|}{F(\log r)}=0 \text { or } \infty \text { a.s. }
$$

depending on whether the integral

$$
\int^{\infty} d x / F(x)
$$

converges or diverges. It remains to note that the last condition is equivalent to the assumption of Corollary 1 for $f(x) \stackrel{\text { def }}{=} F(\log x) / \log x$.

Now we can prove the following theorem on the upper-lower functions of the first type for the Brownian winding process.

Theorem 2. We have

$$
\limsup _{t} \frac{|\Phi(t)|}{\log t f(t)}=0 \text { or } \infty \quad \text { a.s. }
$$

depending on whether the integral

$$
\int_{0}^{\infty} \frac{d x}{x \log x f(x)}
$$

converges or diverges.

Proof. Using relations (3)-(6), we get

$$
\begin{gathered}
\Phi(t)=\gamma^{*}\left(C_{t}\right), \\
\Phi(T(r))=\gamma^{*}\left(\sigma_{\log r}\right),
\end{gathered}
$$

where $\gamma^{*}$ is the standard linear Wiener process and $\sigma_{r}=C_{T(\exp r)}$ denotes the first moment of hitting the point by another Wiener process independent of $\gamma^{*}$. Using Corollary 1 , we get the required statement for the special case where $t \rightarrow \infty$ along the set of moments $T(R)=\min \left\{t>0:\left\|Z_{t}\right\|=R\right\}, R>0$.

To complete the proof, consider a sequence of Markov moments $t_{k}$ such that the variables $\frac{\left|\Phi\left(t_{k}\right)\right|}{\log t_{k} f\left(t_{k}\right)}$ are "small" (respectively, "large") compared with the "close" moments $T(R)$ and use (as in the proof of Corollary 1) the independence of the variables $\operatorname{sign}\left(\Phi(T(R))-\Phi\left(t_{k}\right)\right)$ from the $\sigma$-algebras generated by the events happening before the moments $t_{k}$.

Now we proceed to the study of the functional

$$
\Phi_{1}(t)=\int_{0}^{C_{t}} \mathbb{I}\left(\tilde{\beta}_{s} \in\left[\log \varepsilon_{1}, \log \varepsilon_{2}\right]\right) d \tilde{\gamma}_{s}
$$

Lemma 5. We have

$$
\mathbf{P}\left(\sup _{0 \leq s \leq l_{0}^{\sigma \tau}} w(s) \geqslant y\right)=e^{-y / \sqrt{\tau}}
$$

Proof. Let us use relation 6.3 .3 of [1]:

$$
\frac{d}{d y} \mathbf{P}\left(l_{0}^{\sigma_{r}}<y\right)=\frac{e^{-y / 2 r}}{2 r} .
$$


We have

$$
\begin{aligned}
& \mathbf{P}\left(\sup _{0 \leq s \leq l_{0}^{\sigma r}} w(s) \geqslant y\right)=\frac{1}{2 r} \int_{0}^{\infty} \operatorname{Erfc}(y / \sqrt{2 t}) e^{-t / 2 r} d t \\
& =\frac{1}{r \sqrt{\pi}} \int_{0}^{\infty} e^{-t / 2 r} d t \int_{y / \sqrt{2 t}}^{\infty} e^{-v^{2}} d v \\
& =\frac{1}{r \sqrt{\pi}} \int_{0}^{\infty} e^{-v^{2}} d v \int_{y^{2} / 2 v^{2}}^{\infty} e^{-t / 2 r} d t \\
& =\frac{2}{\sqrt{\pi}} \int_{0}^{\infty} e^{-\left(v^{2}+y^{2} / 4 r v^{2}\right)} d v=e^{-y / \sqrt{r}} .
\end{aligned}
$$

(The last integral is tabulated in $[7,860.25]$. )

Lemma 6. We have

$$
\underset{r}{\limsup } \frac{1}{\sqrt{r} \log \log r}\left|w\left(l_{0}^{\sigma_{r}}\right)\right|=1 \quad \text { a.s. }
$$

Proof. By Lemma 5, for all $\alpha$ greater than one, the series

$$
\sum_{k} P\left(\sup _{0 \leq s \leq l_{0}^{\sigma \alpha k}} w(s) \geqslant F\left(\alpha^{2 k}\right)\right)
$$

converges if

$$
F_{1}(x)=\sqrt{x}\left(\log \frac{\log x}{2}+2 \log \log \frac{\log x}{2}\right)
$$

and diverges if

$$
F_{2}(x)=\sqrt{x} \log \frac{\log x}{2} .
$$

Now we can use Corollary 1. Certainly,

$$
F_{1}(x) / F_{2}(x) \rightarrow 1 \text { and } F_{1}(x) / \sqrt{x} \log \log x \rightarrow 1, \quad x \rightarrow \infty .
$$

Corollary 3. We have

$$
\underset{r}{\limsup } \frac{1}{\sqrt{\log r} \log \log \log r}\left|w\left(\tau_{1}\left(C_{T(r)}\right)\right)\right|=\sqrt{\log \left(\varepsilon_{2} / \varepsilon_{1}\right)} \quad \text { a.s. }
$$

Proof. It suffices to note that

$$
\lim _{r \rightarrow \infty} \tau_{1}(r) / l_{0}^{r}=\log \left(\varepsilon_{2} / \varepsilon_{1}\right)
$$

(see $\left[10\right.$, p. 229]) and to use Lemma 6 and the fact that $C_{T(r)}=\sigma_{\log r}$ and that if $t / t_{1} \rightarrow c>0$ as $t_{1} \rightarrow \infty$, then

$$
\underset{t}{\limsup }|w(t)| / \underset{t_{1}}{\limsup }\left|w\left(t_{1}\right)\right| \rightarrow \sqrt{c} \text { a.s. }
$$

Finally, arguing as in the proof of Theorem 2, we derive from Corollary 3 one more result about the upper-lower functions for winding functionals. 
Theorem 3. We have

$$
\limsup _{t} \frac{\left|\Phi_{1}(t)\right|}{\sqrt{\log t} \log \log \log t}=\sqrt{\log \left(\varepsilon_{2} / \varepsilon_{1}\right)} \quad \text { a.s. }
$$

Unfortunately, no full characterization of upper-lower functions similar to Theorems 2 and 3 is known for functions of the second type and even for functions of the first type for $\Phi_{2}$. Nevertheless, some estimates can be obtained by our method.

Lemma 7. (a) Let $l_{0}^{\sigma_{r}}$ be the local time at zero of the standard linear Wiener process up to its hitting time $\sigma_{r}$ of the point $r, r>0$. Let

$$
F(y) / y \log \frac{G(y)}{y} \rightarrow \infty
$$

where $G$ is an increasing function satisfying conditions $\int^{\infty} d y G(y)^{-1}<\infty, G(y) / y$ is also increasing, and for every $\alpha$ greater than one we have

$$
\underset{y}{\lim \sup } G(\alpha y) / G(y)<\infty
$$

Then

$$
\underset{r \rightarrow \infty}{\limsup } l_{0}^{\sigma r} / F(r)=0 \text { a.s. }
$$

(b) Let $F$ be an increasing function such that $F(y) / y$ is decreasing and

$$
\int^{\infty} d y F(y) / y^{2}<\infty ; \quad \limsup _{y} F(\alpha y) / F(y)<\infty, \forall \alpha>1
$$

Then

$$
\liminf _{r \rightarrow \infty} l_{0}^{\sigma_{r}} / F(r)=\infty \quad \text { a.s. }
$$

Proof. Be relation 6.3 .3 of [1], we have

$$
\mathbf{P}\left(l_{0}^{\sigma_{r}}>y\right)=e^{-y / 2 r}
$$

i.e.,

$$
\mathbf{P}\left(l_{0}^{\sigma_{r}}<y\right)=O(y / 2 r), \quad y / r \rightarrow 0 .
$$

Fix an $\alpha$ greater than one and define $R_{k}=\alpha^{k}, k \in \mathbb{N}$.

To prove assertion (b), note that for $c>0$ we have

$$
\sum_{k} \mathrm{P}\left(l_{0}^{\sigma_{R_{k}}}<c F\left(R_{k}\right)\right)=\sum_{k} O\left(F\left(\alpha^{k}\right) / \alpha^{k}\right)<\infty
$$

if and only if

$$
\int^{\infty} d x F\left(\alpha^{x}\right) / \alpha^{x}=\frac{1}{\log \alpha} \int^{\infty} d y F(y) / y^{2}<\infty .
$$

By the Borel-Cantelli lemma and in view of the free choice of $c>0$, we get

$$
\liminf _{k} l_{0}^{\sigma_{\alpha^{k}}} / F\left(\alpha^{k}\right)=\infty
$$


if

$$
\int^{\infty} d y F(y) / y^{2}<\infty
$$

It remains to use the regularity of the function $F$ and the monotonicity of $l_{0}^{\sigma_{r}}$. Assertion (b) is proved. To prove assertion (a), note that

$$
\sum_{k} \mathrm{P}\left(l_{0}^{\sigma_{\alpha^{k}}}>c F\left(\alpha^{k}\right)\right)=\sum_{k} \exp \left(-\frac{c F\left(\alpha^{k}\right)}{2 \alpha^{k}}\right)<\infty
$$

if and only if

$$
\int^{\infty} d x \exp \left(-\frac{c F\left(\alpha^{x}\right)}{2 \alpha^{z}}\right)=\frac{1}{\log \alpha} \int_{0}^{\infty} \frac{d y}{y \exp (c F(y) / 2 y)}<\infty .
$$

Simple monotonicity arguments complete the proof.

Corollary 4. (a) Let

$$
F(y) / y \log \frac{G(y)}{y} \rightarrow \infty,
$$

where $G$ is such that $\int^{\infty} d y G(y)^{-1}<\infty, G(y) / y$ is increasing, and for any $\alpha$ greater that one we have

$$
\underset{y}{\lim \sup } G(\alpha y) / G(y)<\infty
$$

Then

$$
\underset{r \rightarrow \infty}{\limsup } \tau_{1}\left(\sigma_{r}\right) / F(r)=0 \quad \text { and } \quad \lim \sup t \rightarrow \infty \tau_{1}\left(C_{t}\right) / F(\log t)=0 \quad \text { a.s. }
$$

(b) Let $F$ be an increasing function such that $F(y) / y$ is decreasing and

$$
\int^{\infty} d y F(y) / y^{2}<\infty, \quad \underset{y}{\lim \sup } F(\alpha y) / F(y)<\infty, \forall \alpha>1
$$

Then

$$
\underset{r \rightarrow \infty}{\liminf } \tau_{1}\left(\sigma_{r}\right) / F(r)=\infty \quad \text { and } \quad \liminf t \rightarrow \infty \tau_{1}\left(C_{t}\right) / F(\log t)=\infty \text { a.s. }
$$

Proof. It suffices to recall that

$$
\lim _{r \rightarrow \infty} \tau_{1}(r) / l_{0}^{r}=\log \left(\varepsilon_{2} / \varepsilon_{1}\right) \text { a.s. }
$$

(we have already used this when proving Corollary 3). Furthermore, $C_{T(r)}=\sigma_{\log r}$ and

$$
\frac{\log T(r)}{2 \log r} \rightarrow 1, \quad r \rightarrow \infty
$$

Now let us investigate the lower bound of the modulus of $\Phi_{1}$. 
Theorem 4. Let $F$ be an increasing function such that $F(y) / y$ is decreasing and

$$
\int^{\infty} d y F(y) / y^{2}<\infty, \quad \underset{y}{\lim \sup } F(\alpha y) / F(y)<\infty, \forall \alpha>1
$$

Then

$$
\liminf _{t} \sqrt{\frac{\log \log F(\log t)}{F(\log t)}} \sup _{0 \leq s \leq t}\left|\Phi_{1}(t)\right|=\infty \text { a.s. }
$$

We need the following result, which "complements" in a certain sense the standard LIL for the onedimensional Wiener process (see [9, Theorem 11.6]).

Theorem (Fristedt). Let $\gamma$ be the standard linear Wiener process. Then

$$
0<\liminf _{t} \sqrt{\frac{\log \log t}{t}} \sup _{0 \leq s \leq t}|\gamma(t)|<\infty \text { a.s. }
$$

Proof of Theorem 4. By Lemma 7 and relations (16") and (18), we have

$$
\begin{gathered}
\liminf _{t} \sqrt{\frac{\log \log F(\log t)}{F(\log t)}} \sup _{0 \leq s \leq t}\left|\Phi_{1}(t)\right|=\liminf _{t} \sqrt{\frac{\log \log F(\log t)}{F(\log t)}} \sup _{0 \leq s \leq t}\left|\gamma^{*}\left(\tau_{1}\left(C_{t}\right)\right)\right| \\
\geqslant \liminf _{t} \frac{\sup _{0 \leq s \leq t}\left|\gamma^{*}\left(\tau_{1}\left(C_{t}\right)\right)\right|}{\sqrt{\tau_{1}\left(C_{t}\right) / \log \log \tau_{1}\left(C_{t}\right)}} \cdot \liminf _{t} \frac{\sqrt{\tau_{1}\left(C_{t}\right) / \log \log \tau_{1}\left(C_{t}\right)}}{\sqrt{F(\log t) / \log \log F(\log t)}}=\infty
\end{gathered}
$$

according to the lemma. The theorem is proved.

The following result can be proved in a similar way.

Theorem 5. Let

$$
F(y) / y \log \frac{G(y)}{y} \rightarrow \infty
$$

where $G$ is such that $\int_{0}^{\infty} d y G(y)^{-1}<\infty, G(y) / y$ and $F(y) / y$ are increasing, and

$$
\underset{y}{\limsup } G(e y) / G(y)<\infty
$$

Then

$$
\liminf _{t} \frac{\sup _{0 \leq s \leq t}\left|\Phi_{1}(t)\right|}{\sqrt{F(\log t) / \log \log F(\log t)}}=0 \text { a.s. }
$$

Proof. In view of $\left(16^{\prime}\right)$, we have

$$
\begin{aligned}
& \liminf _{t} \frac{\sup _{0 \leq s \leq t}\left|\Phi_{1}(t)\right|}{\sqrt{F(\log t) / \log \log F(\log t)}}=\liminf _{t} \frac{\sup _{0 \leq s \leq t}\left|\gamma^{*}\left(\tau_{1}\left(C_{t}\right)\right)\right|}{\sqrt{F(\log t) / \log \log F(\log t)}} \\
\leqslant & \liminf _{t} \frac{\sup _{0 \leq s \leq t}\left|\gamma^{*}\left(\tau_{1}\left(C_{t}\right)\right)\right|}{\sqrt{\tau_{1}\left(C_{t}\right) / \log \log \tau_{1}\left(C_{t}\right)}} \times \limsup \frac{\sqrt{\tau_{1}\left(C_{t}\right) / \log \log \tau_{1}\left(C_{t}\right)}}{\sqrt{F(\log t) / \log \log F(\log t)}}=0 .
\end{aligned}
$$

In conclusion, we present the complete list of results obtained by the author. 
Theorem. Let $G_{i}, F_{i}, i=1,2$, be monotone increasing functions such that $F_{2}(y) / y^{2}$ is monotone decreasing, $F_{1}(y) / y \log \frac{G(y)}{y}$ and $G_{1}(y) / y$ are monotone increasing and tend to infinity, and for any $\alpha$ greater than one we have

$$
\underset{t}{\limsup } F_{1,2}(\alpha y) / F_{1,2}(y)<\infty, \quad \underset{t}{\limsup } G_{1,2}(\alpha y) / G_{1,2}(y)<\infty
$$

and

$$
\int^{\infty} d y F_{2}(y) / y^{2}<\infty, \quad \int_{0}^{\infty}\left(G_{1}(y)\right)^{-1} d y<\infty
$$

Then

(i) we have a.s.

$$
\begin{gathered}
\limsup _{t} \frac{\left|\Phi_{1}(t)\right|}{\sqrt{\log t} \log \log \log t}=\sqrt{\log \left(\varepsilon_{2} / \varepsilon_{1}\right)}, \\
\liminf _{t} \sqrt{\frac{\log \log F_{1}(\log t)}{F_{1}(\log t)}} \sup _{0 \leq s \leq t}\left|\Phi_{1}(t)\right|=0 \\
\liminf _{t} \sqrt{\frac{\log \log F_{2}(\log t)}{F_{2}(\log t)}} \sup _{0 \leq s \leq t}\left|\Phi_{1}(t)\right|=\infty
\end{gathered}
$$

(ii) we have a.s.

$$
\begin{aligned}
\limsup \frac{\left|\Phi_{2}(t)\right|}{G_{1}(\log t) \sqrt{\log \log \log t}} & =0, \\
\limsup \frac{\log ^{2} \log t}{\log t}\left|\Phi_{2}(t)\right| & =\infty, \\
\liminf _{t} \frac{\sqrt{\log \log \log t}}{G_{1}(\log t)} \sup _{0 \leq s \leq t}\left|\Phi_{2}(t)\right| & =0, \\
\liminf _{t} \frac{(\log \log t)^{2} \log \log \log t}{\log t} \sup _{0 \leq \Delta \leq t}\left|\Phi_{2}(t)\right| & =\infty ;
\end{aligned}
$$

(iii) we have a.s. $\lim \sup _{t} \frac{|\Phi(t)|}{\log t G_{2}(t)}=0$ or $\infty$, depending on whether $\int_{.}^{\infty} \frac{d x}{x \log x G_{2}(x)}$ converges or diverges,

$$
\begin{aligned}
& \liminf _{t} \frac{\sqrt{\log \log \log t}}{G_{1}(\log t)} \sup _{0 \leq s \leq t}|\Phi(t)|=0, \\
& \liminf _{t} \frac{\log \log \log t}{\log t} \sup _{0 \leq s \leq t}|\Phi(t)|=\infty .
\end{aligned}
$$

Thereafter, the author succeeded in proving that

$$
\limsup _{t} \frac{\left|\Phi_{2}(t)\right|}{\log t \log \log \log t}=\frac{\pi}{2} \text { a.s. }
$$

The research was partially supported by an AMS-fSU' 93 grant.

Translated by E. A. Dorofeev. 


\section{REFERENCES}

1. P. E. Baskakova and A. N. Borodin, "Tables of distributions of the functionals of Brownian motion," Zap. Nauchn. Semin. POMI, 194, 8-20 (1992).

2. C. Bélisle, "Windings of random walks," Ann. Probab., 17, 1377-1402 (1989).

3. C. Bélisle, "Windings of spherically symmetric random walks via Brownian embedding," Stat. Probab. Lett., 12, 345-349 (1991).

4. J. Bertoin and W. Werner, "Comportement asymptotique du nombre de tours effectués par la trajectoire brownienne plane," Lect. Notes Math. (to appear).

5. K. Burdzy, J. Pitman, and M. Yor, "Some asymptotic laws for crossings and excursions," Astérisque, 157-158, 59-74 (1988).

6. K. Burdzy, J. Pitman, and M. Yor, "Brownian crossings between spheres," J. Math. Anal. Appl., 148, 101-120 (1990).

7. K. E. Dambis, "On decomposition of continuous submartingales," Teor. Veroyatn. Primen., 10, 438-448 (1965).

8. W. Feller, An Introduction to Probability Theory and Its Applications, Wiley, New York (1969).

9. B. Fristedt, "Sample functions of stochastic processes with stationary, independent increments," $A d v$. Probab., 3, 271-396 (1974).

10. R. Itô and H. P. J. McKean, Diffusion Processes and Their Sample Paths, Springer, Berlin (1965).

11. B. Jamison, "Reciprocal processes; the stationary Gaussian case," Ann. Math. Stat., 41, 1624-1630 (1970).

12. F. B. Knight, "A reduction of continuous square-integrable martingales to Brownian motion," Lect. Notes Math., 190, 19-31 (1971).

13. F. B. Knight, "On integrability of martingale time changes," Progress Probab. Stat., 15, 193-221 (1988).

14. F. B. Knight, "Some remarks on mutual windings," Preprint Univ. Illinois, Urbana (1992).

15. T. Lyons and H. P. McKean, "Windings of the plane Brownian motion," Adv. Math., 51, 212-225 (1984).

16. P. Messulam and M. Yor, "On D. Williams' 'pinching method' and some applications," J. London Math. Soc., 2, 348-364 (1982).

17. J. Pitman and M. Yor, "Asymptotic laws of planar Brownian motion," Ann. Probab., 14, 733-779 (1986).

18. J. Pitman and M. Yor, "Further asymptotic laws of planar Brownian motion," Ann. Probab., 17, 9651011 (1989).

19. D. Revuz and M. Yor, Continuous Martingales and Brownian Motion, Springer-Verlag, New York (1991).

20. Z. Shi, "Liminf behaviors of the windings and Lévy's stochastic areas of planar Brownian motion," Preprint Univ. Paris-VI, Paris (1993).

21. F. Spitzer, "Some theorems concerning two-dimensional Brownian motion," Trans. Am. Math. Soc., 87, 187-197 (1958).

22. A. N. Shiryaev, Probability [in Russian], Nauka, Moscow (1989).

23. M. Yor, "Études asymptotiques des nombres de tours de plusieurs mouvements browniens correlés," Progress Probab. Stat., 28, 441-455 (1991). 